\title{
Effect of a nitric oxide synthase inhibitor and a CXC chemokine receptor-4 antagonist on tumor growth and metastasis in a xenotransplanted mouse model of adenoid cystic carcinoma of the oral floor
}

\author{
KAZUKI TAKAOKA $^{1 *}$, SAYAKA HIDAKA ${ }^{1 *}$, SUSUMU HASHITANI $^{1}$, EMI SEGAWA ${ }^{1}$, \\ MICHIYO YAMAMURA ${ }^{1}$, NORIAKI TANAKA ${ }^{2}$, YUSUKE ZUSHI ${ }^{1}$, KAZUMA NOGUCHI $^{1}$, \\ HIROMITSU KISHIMOTO $^{1}$ and MASAHIRO URADE ${ }^{1}$ \\ ${ }^{1}$ Department of Oral and Maxillofacial Surgery, Hyogo College of Medicine, Nishinomiya, Hyogo 663-8501; \\ ${ }^{2}$ The First Department of Oral and Maxillofacial Surgery, Osaka University Graduate School of Dentistry, Suita, Osaka, Japan
}

Received April 4, 2013; Accepted May 17, 2013

DOI: $10.3892 /$ ijo.2013.2011

\begin{abstract}
Nitric oxide (NO) is related to angiogenesis and tumor progression and chemokine receptor-4 (CXCR4) plays a central role in cell migration in metastasis and dissemination of cancer. The present study evaluated the effectiveness of a NOS inhibitor and a CXCR4 antagonist, given as single agents or in combination, in a xenotransplanted mouse model of adenoid cystic carcinoma (ACC) of the oral floor. A metastatic tumor (ACCIM) derived from a cervical metastatic lesion of human ACC that was transplantable in nude mice was used. ACCIM showed a high frequency of spontaneous metastasis to the lung when transplanted subcutaneously in nude mice. Mice with subcutaneous transplants of ACCIM were subdivided into six groups and intraperitoneally received one of the following treatments daily for 5 weeks: a) PBS (control), b) AMD3100 (CXCR4 antagonist), c) L-NAME (NOS inhibitor), d) 1400W (iNOS inhibitor), e) both AMD3100 and L-NAME (AMD3100+L-NAME) and f) both AMD3100 and 1400W (AMD3100+1400W). Tumor growth was evaluated during treatment and metastasis was assessed at 28 weeks. Single-agent treatment with AMD3100, L-NAME or $1400 \mathrm{~W}$ inhibited tumor growth by $20.8,26.5$ and $54.5 \%$, respectively. Combined treatment with AMD3100+L-NAME and AMD3100+1400W inhibited tumor growth remarkably by 48.0 and $50.2 \%$, respectively. Immunohistochemical analysis
\end{abstract}

Correspondence to: Dr Kazuki Takaoka, Department of Oral and Maxillofacial Surgery, Hyogo College of Medicine, 1-1 Mukogawa-cho, Nishinomiya, Hyogo 663-8501, Japan

E-mail:ktaka@hyo-med.ac.jp

*Contributed equally

Key words: nitric oxide synthase inhibitor, chemokine receptor-4 antagonist, adenoid cystic carcinoma, oral floor, tumor growth, metastasis revealed lower expression of CXCR4, iNOS and eNOS in tumor cells treated with AMD3100+L-NAME or AMD3100+1400W compared to control tumor cells and increased numbers of apoptotic tumor cells were demonstrated using the TUNEL method. CXCR4 expression decreased in 1400W-treated tumors using western blot analysis. When the effect of each agent on tumor-induced angiogenesis in tumor stroma was examined histologically, microvessel density was significantly lower in the groups treated with 1400W, AMD3100+L-NAME or AMD3100+1400W compared to the control, AMD3100 and L-NAME groups. Moreover, treatment with AMD3100 or 1400W markedly inhibited lung metastasis. Our results indicated that single-agent treatment with $1400 \mathrm{~W}$ and combined treatment with AMD3100+L-NAME or AMD3100+1400W induced apoptosis and significantly inhibited tumor-induced angiogenesis and proliferation of ACCIM in vivo. Blockade of CXCR4 and iNOS was suggested to inhibit lung metastases from ACCIM. CXCR4 and iNOS may, thus, be important prognostic factors for long-term survival in ACC.

\section{Introduction}

Adenoid cystic carcinoma (ACC) is a generally slow-growing, but highly malignant salivary gland neoplasm with remarkable capacity for invasion and metastasis. Patients with ACC of the salivary glands have a fair 5-year survival rate, but long-term overall survival (10-20 years) remains poor $(1,2)$ owing to the development of late recurrence or metastases, even with low-grade tumors $(1,2)$. Distant metastases (to lung, bone and soft tissues) develop in 40-60\% of patients, despite good local tumor control. Better control of lung metastases in particular is expected to improve outcomes in patients with ACC.

Nitric oxide (NO) is a multifunctional gaseous molecule synthesized from L-arginine by NO synthase (NOS). There are three isoforms of NOS: neuronal NOS (nNOS), endothelial NOS (eNOS) and inducible isoform of NOS (iNOS). nNOS and eNOS are constitutively expressed and are thus referred to as constitutive NOS. In contrast, iNOS is transcriptionally 
regulated and induced by inflammatory cytokines, endotoxins, hypoxia and oxidative stress $(3,4)$. iNOS produces high, sustained concentrations of NO, whereas the other two isoforms produce low, transient concentrations of NO (5). Recent studies have shown positive correlations between iNOS and poor outcomes in patients with breast cancer and melanoma $(6,7)$. These observations suggest that NO generated by iNOS has multiple physiologic and pathologic effects. Other recent studies report that eNOS can modulate cancer-related events, such as angiogenesis, apoptosis, cell cycle dynamics, tumor invasion and metastasis (8). We previously confirmed the antitumor effects of NOS inhibitor and iNOS inhibitor against a human KB carcinoma cell line in which malignancy was increased by gene transfer of COX-2 cDNA (9).

CXCR4 is a receptor for stromal cell-derived factor-1 $\alpha$ (SDF-1 $\alpha$; the so called CXCL12), a chemokine expressed in several tissues and organs, including skin, lymph nodes, lung, liver and bone marrow (10-13). SDF-1 $\alpha$ stimulates cell adhesion, migration and activation (13-18). CXCR4 is expressed in different tumor cell lines $(10,19)$ and the pulmonary metastatic potential of cells expressing CXCR4 was higher than that of their CXCR4-negative counterparts in a B16 murine melanoma model $(20,21)$. Furthermore, CXCR4 has been shown to play a key role in metastases from breast cancer and melanoma $(10,22)$. We previously suggested that CXCR4 expression is closely related to metastatic potential in surgical specimens of ACC (23).

We established a new human tumor cell line (ACCI) derived from $\mathrm{ACC}$ of the oral floor. ACCI shows a cribriform pattern histologically and is serially transplantable into nude mice. This tumor is associated with spontaneous metastasis to the neck at the second passage level and the histological features change from ACC to undifferentiated carcinoma. The metastatic tumor, designated as ACCIM, shows a high frequency of spontaneous metastasis to the lung when transplanted subcutaneously in nude mice (24).

As mentioned above, $\mathrm{NO}$ is related to apoptosis, angiogenesis and metastasis and CXCR4 plays a central role in cell migration in metastases from cancer. The present study evaluated the effectiveness of a NOS inhibitor and a CXCR4 antagonist, given as single agents or in combination, in a xenotransplanted mouse model of ACCIM.

\section{Materials and methods}

Animals. Five-week-old female nude mice (BALB/c nu/nu; Oriental Yeast Ltd., Tokyo, Japan) were used as experimental animals. Food was supplied ad libitum and the animals were housed under sterile conditions. All animal experiments were performed in compliance with the Guidelines for Experimental Animals of Hyogo College of Medicine.

Agents. $\mathrm{N}^{\mathrm{G}}$-nitro-L-arginine-methyl ester (L-NAME), a NOS inhibitor and 1,1'-\{1,4-phenylenebis(methylene)\}bis-1,4,8,11tetraazacyclotetradecane octahydrochloride (AMD3100), a CXCR4 antagonist, were purchased from Sigma-Aldrich Co. (St. Louis, MO, USA). Dihydrochloride (1400W), a selective iNOS inhibitor, was purchased from Cayman Chemical Co. (Ann Arbor, MI, USA). These agents were dissolved in $\mathrm{Mg}^{2+}$ and $\mathrm{Ca}^{2+}$-free phosphate-buffered saline (PBS[-]) before use.

\section{Scheme of experiment}

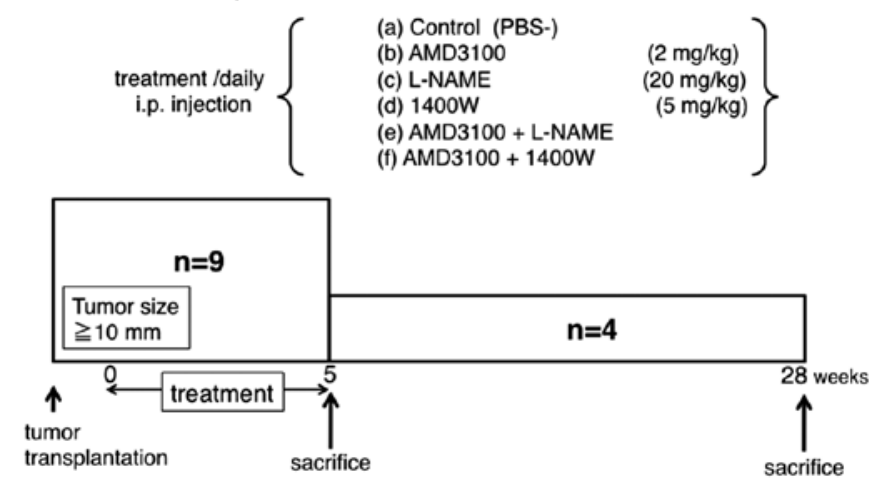

Figure 1. Scheme of experiment. ACCIM was cut into $\sim 2 x 2-\mathrm{mm}$ pieces and implanted subcutaneously in nude mice. When the tumor reached $10 \mathrm{~mm}$ in diameter, mice were subdivided into six groups and were assigned to receive one of the following treatments by intraperitoneal injection every day for 5 weeks: (a) vehicle (PBS[-]), (b) AMD3100 (2 mg/kg), (c) L-NAME (20 mg/ $\mathrm{kg}$ ), (d) $1400 \mathrm{~W}(5 \mathrm{mg} / \mathrm{kg}$ ), (e) both AMD3100 and L-NAME (AMD3100+LNAME), or (f) both AMD3100 and 1400W (AMD3100+1400W). Five and 28 weeks after the start of treatment, 5 and 4 mice from each group were randomly selected and sacrificed, respectively.

Tumor tissue and heterotransplantation into nude mice. The original tumor tissue was obtained from the surgical specimens of a 72-year-old man with ACC of the oral floor. Histologically, the tumor showed a cribriform pattern. Metastatic tumor tissue was obtained from a lesion of spontaneous neck metastasis from the above ACC transplanted into a second passage of nude mice. The tumor was rinsed 3 times in PBS, cut into $\sim 2 \times 2-\mathrm{mm}$ pieces and transplanted into the flanks of the mice. At the second passage level, $\sim 1$ year after ACC transplantation, another tumor mass appeared in the neck, apart from the transplanted site of the tissue fragment. Although this tumor was an undifferentiated carcinoma histologically, it was considered a metastatic lesion and designated as ACCIM. ACCIM produced multiple metastases to lymph nodes and lungs 5 months after transplantation (24).

Treatment of agents on the subcutaneously xenotransplanted tumor of ACCIM in nude mice. ACCIM was cut into $\sim 2 \times 2-\mathrm{mm}$ pieces and implanted subcutaneously in nude mice. Approximately 19 days after ACCIM xenotransplantation, when the tumor reached $10 \mathrm{~mm}$ in diameter, mice were subdivided into six groups and were assigned to receive one of the following treatments by intraperitoneal injection every day for 5 weeks: a) vehicle (PBS[-]), b) AMD3100 (2 mg/ $\mathrm{kg})$, c) L-NAME $(20 \mathrm{mg} / \mathrm{kg})$, d) $1400 \mathrm{~W}(5 \mathrm{mg} / \mathrm{kg})$, e) both AMD3100 and L-NAME (AMD3100+L-NAME), or f) both AMD3100 and 1400W (AMD3100+1400W). Five weeks and 28 weeks after the start of treatment, 5 and 4 mice from each group were randomly selected and sacrificed, respectively (Fig. 1). The tumor volume was calculated by the following formula: volume $\left(\mathrm{mm}^{3}\right)=a^{2} x b / 2$, where $a$ is the tumor width in $\mathrm{mm}$ and $b$ is the tumor length in $\mathrm{mm}$ (25). Necropsies were performed to identify macro-metastases to the lung 28 weeks after treatment began. The primary tumors and lungs were harvested, fixed in $10 \%$ formalin, embedded in paraffin, cut into $4-\mu \mathrm{m}$-thick sections and stained with hematoxylin and eosin (H\&E) according to conventional procedures. 

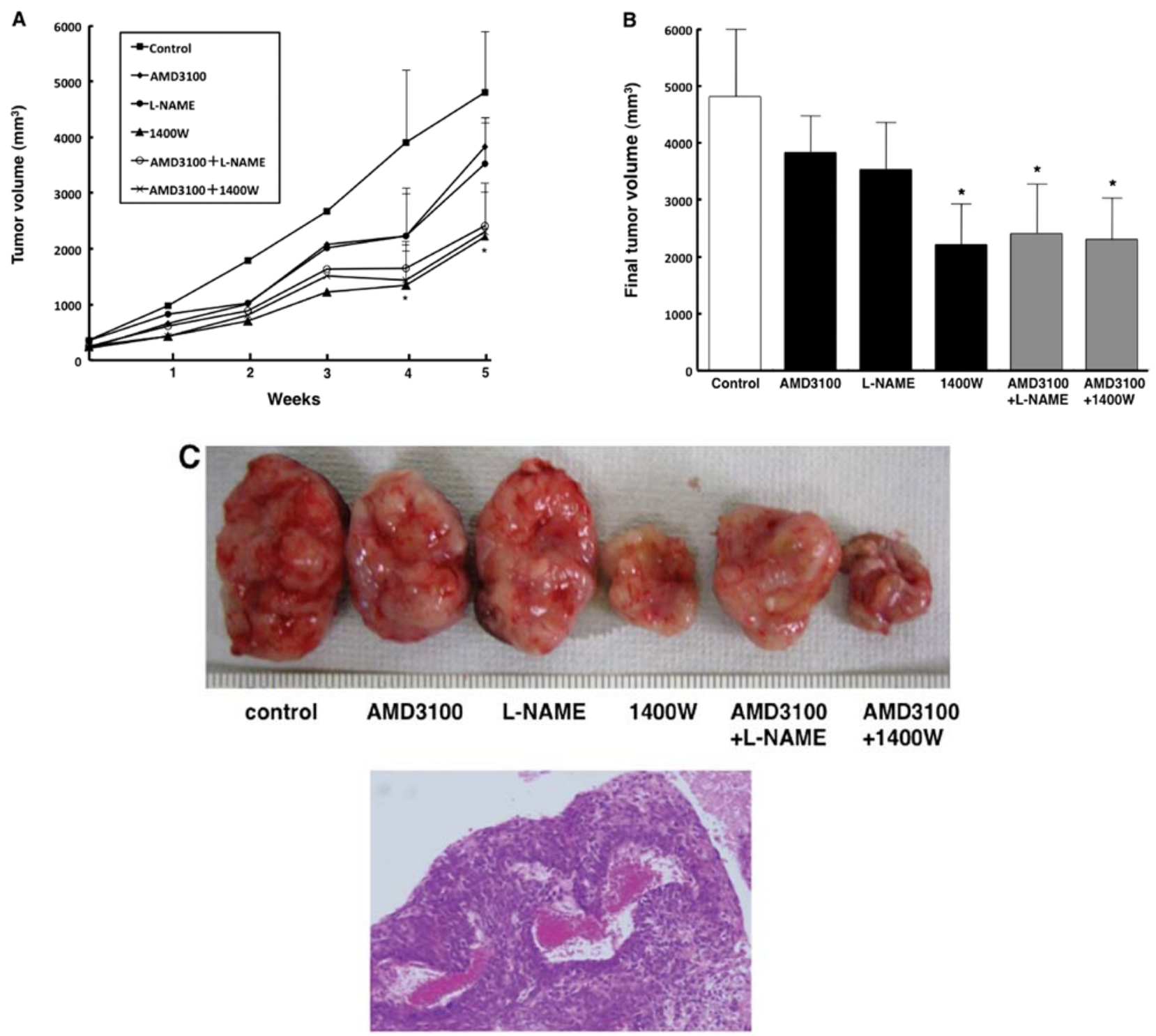

H\&E

Figure 2. Effect of agents on subcutaneously xenotransplanted tumor volume. Tumors developing at the inoculated sites were measured with calipers once a week. The relative tumor weight was determined according to the method of Battelle Columbus Laboratories (25). (A) $\mathbf{\bullet}$, control; $\bullet$, AMD3100; $\bullet$, L-NAME;

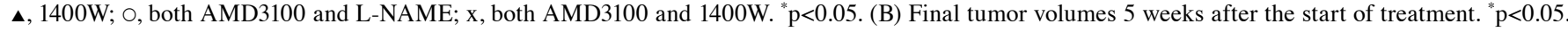
(C) Macroscopic and micrographic features of tumor specimens obtained at the end of the observation period (H\&E stain; original magnification, $\mathrm{x} 100)$.

Immunohistochemical study of xenotransplanted tumors. Immunohistochemical examination was performed with the use of the avidin-biotin-peroxidase complex (ABC) staining method (26). Briefly, endogenous peroxidase activity was blocked in tissue specimens by treatment with $0.3 \% \mathrm{H}_{2} \mathrm{O}_{2}$ in methanol for $5 \mathrm{~min}$. The specimens were washed and treated with $1 \%$ normal horse serum in PBS for $15 \mathrm{~min}$. After washing with PBS, rabbit polyclonal antibody for human CXCR4 (Abcam, Cambridge, UK), rabbit polyclonal antibody for human NOS3 (Santa Cruz Biotechnology, Santa Cruz, CA, USA), or rabbit polyclonal antibody for human NOS2 (Santa Cruz), was applied as primary antibody at $4^{\circ} \mathrm{C}$ overnight. After further washing with PBS, the specimens were incubated with ABC complex solution (Vectastain, Vector Lab., Burlingame, CA, USA) at room temperature for $15 \mathrm{~min}$. After washing with PBS, biotinylated goat anti-mouse $\operatorname{IgG}$ (Vector) was applied to the sections, which were then incubated for $30 \mathrm{~min}$ at room temperature. The specimens were treated for $\sim 5$ min with a substrate solution containing 3,3'-diaminobenzidine tetrahydrochloride (Wako Pure Chemical Industries, Ltd., Osaka, Japan) and $\mathrm{H}_{2} \mathrm{O}_{2}$. Finally, the specimens were counterstained with hematoxylin, dehydrated and mounted with glycerol gelatin.

The CXCR4, iNOS and eNOS labeling indexes (LI) were obtained by calculating the ratio of positive cells to the total number of tumor cells counted in well-labeled areas, as determined by scanning twenty areas at x200 magnification.

Western blot analysis. Tumor samples were lysed in a lysis buffer consisting of PBS[-], supplemented with $20 \mathrm{mM}$ Tris-HCl, $\mathrm{pH}$ 8.0, $1 \%$ NP40, $150 \mathrm{mM} \mathrm{NaCl}, 1 \mathrm{mM}$ EDTA, $10 \%$ glycerol, $0.1 \% \beta$-mercaptoethanol, $0.5 \mathrm{mM}$ dithiothreitol 
and a mixture of proteinase inhibitors consisting of $1 \mathrm{mM}$ phenylmethylsulfonyl fluoride, $10 \mu \mathrm{g} / \mathrm{ml}$ aprotinin, $5 \mu \mathrm{g} / \mathrm{ml}$ leupeptin, $5 \mathrm{mM}$ benzamidine, $1 \mu \mathrm{g} / \mathrm{ml}$ pepstatin, $2 \mu \mathrm{g} / \mathrm{ml}$ antipain hydrochloride (Boehringer, Mannheim, Germany), $50 \mu \mathrm{M}$ 4-(2-aminoethyl)-benzenesulfonyl fluoride hydrochloride (Wako Pure Chemical Industries), $2 \mathrm{mM}$ sodium orthovanadate (Sigma-Aldrich) and $20 \mathrm{U} / \mathrm{ml}$ ulinastatin (Mochida Pharmaceutical, Tokyo, Japan). Lysates containing $15 \mu \mathrm{g}$ protein were subjected to electrophoresis in a $10-20 \%$ gradient SDS-PAGE mini gel (Bio-Rad, Chicago, IL, USA) and blotted onto a PVDF membrane using Multiphor II (Amersham Pharmacia Biotech, Buchinghamshire, UK) for 30 min. The blotted membrane was blocked with $5 \%$ skim milk in $10 \mathrm{mM}$ Tris- $\mathrm{HCl}, \mathrm{pH} \mathrm{7.2,} \mathrm{containing} 150 \mathrm{mM} \mathrm{NaCl}$ and $0.5 \%$ Tween-20 and was incubated with primary antibodies $(0.1-1 \mu \mathrm{g} / \mathrm{ml})$ at $4^{\circ} \mathrm{C}$ for $16 \mathrm{~h}$ as described below. The membrane was then incubated with alkaline phosphataseconjugated secondary antibodies $(0.02 \mu \mathrm{g} / \mathrm{ml})$ for $4 \mathrm{~h}$ at room temperature as described below. The membrane was rinsed and then treated with nitroblue tetrazolium (Sigma-Aldrich) and 5-bromo-4-chloro-3-indolyl phosphate (Sigma-Aldrich) to visualize the protein bands. The primary antibodies used were rabbit polyclonal antibody for human CXCR4 (Abcam) and rabbit polyclonal antibody against iNOS and eNOS (Santa Cruz). The secondary antibodies used were anti-rabbit IgGs conjugated with alkaline phosphatase (Santa Cruz). Actin was used as an internal control.

Assessment of apoptosis in xenotransplanted tumors by agents. To detect DNA breaks, in situ terminal doxynucleotidyl transferase (TdT)-mediated dUTP-digoxigenin nick-end labeling (TUNEL) was performed as described by Gavroieli et al (27). Briefly, after deparaffinization and blocking of endogenous peroxidase with $0.3 \% \mathrm{H}_{2} \mathrm{O}_{2}$ in methanol for $30 \mathrm{~min}$ at room temperature, the sections were treated with $20 \mu \mathrm{g} / \mathrm{ml}$ proteinase K (Dako Cytomention, Glostrup, Denmark) for $15 \mathrm{~min}$ at room temperature. The sections were submitted to TdT reaction in the presence of terminal transferase and biotin-16-dUTP for $60 \mathrm{~min}$ at $37^{\circ} \mathrm{C}$. The sections were then incubated with diluted peroxidase conjugated streptavidin for $30 \mathrm{~min}$ at room temperature to detect biotin-16-dUTP labeling, followed by color development with a solution containing 3,3'-diaminobenzidine and $\mathrm{H}_{2} \mathrm{O}_{2}$. Methyl green was used for counterstaining. TUNEL-positive cancer cells were counted in twenty areas at high magnification $(x 400)$ that show the well-labeled areas.

Assay for microvessel density (MVD). Microvessels were detected by immunohistochemical staining for CD31, a marker for vascular endothelial cells. After pretreatment with $0.25 \%$ trypsin for $10 \mathrm{~min}$, tissue sections were immunostained with an anti-human CD31 mouse monoclonal antibody (Novocastra, Newcastle Upon Tyne, UK) using the SABC method. MVD was determined by counting the number of vessels at magnification (x200) of the tumor stroma that contained the highest number of capillaries.

Statistical analysis. Statistical analysis was done with Student's t-test. Differences were considered statistically significant when the $\mathrm{p}$-value was $<0.05$.

\section{Results}

Effect of agents on the growth of subcutaneously xenotransplanted tumors of ACCIM in nude mice. All agents were well tolerated by the mice, without weight loss or signs of toxicity. These agents inhibited the proliferation of the subcutaneously xenotransplanted tumors. Treatment with $1400 \mathrm{~W}$ for 4 and 5 weeks significantly inhibited tumor growth as compared with treatment with vehicle $(\mathrm{p}<0.05)$ (Fig. 2A). Combined treatment (AMD3100+L-NAME and AMD3100+1400W) also significantly inhibited tumor growth as compared with vehicle at the end of the observation period $(\mathrm{p}<0.05)$. The reduction rate in tumor growth did not differ significantly among these treatments (1400W, AMD3100+L-NAME and AMD3100+1400W). The final mean tumor volume per mouse was $2234 \pm 556 \mathrm{~mm}^{3}(54.5 \%$ decrease $)$ in $1400 \mathrm{~W}$-treated mice, $2554 \pm 612 \mathrm{~mm}^{3}(48.0 \%$ decrease) in AMD3100+LNAME-treated mice and $2443 \pm 602 \mathrm{~mm}^{3}$ (50.2\% decrease) in AMD3100+1400W-treated mice, as compared with $4910 \mathrm{~mm}^{3}$ in vehicle-treated mice (Fig. $2 \mathrm{~B}$ and C). These tumors were undifferentiated carcinoma histologically (Fig. 2C).

Immunohistochemical evaluation. The immunoreactivity of each specimen was evaluated by light and transmission microscopy to assess the intensities of CXCR4, iNOS and eNOS expression. The labeling index (LI) of CXCR4 was significantly lower in tumors treated with AMD3100 (59.8\%) than in tumors treated with vehicle $(89.3 \%)$. In contrast, there were no significantly differences in the LI of CXCR4 in tumors treated with L-NAME or $1400 \mathrm{~W}$ as compared with tumors treated with vehicle (Fig. 3). The LI of iNOS was $83.1 \%$ in vehicle-treated tumors, $79.5 \%$ in L-NAME-treated tumors, $58.0 \%$ in $1400 \mathrm{~W}$-treated tumors and $61.5 \%$ in AMD3100treated tumors. The LI of iNOS was significantly lower in tumors treated with $1400 \mathrm{~W}$ or with AMD3100 than in those treated with vehicle (Fig. 4). The LI of eNOS was $80.1 \%$ in vehicle-treated tumors, $64.5 \%$ in L-NAME-treated tumors, $54.7 \%$ in $1400 \mathrm{~W}$-treated tumors and $75.3 \%$ in AMD3100treated tumors. The LI of eNOS was significantly lower in L-NAME- or $1400 \mathrm{~W}$-treated tumors than in vehicle-treated tumors (Fig. 5). The decreases in the LI of iNOS and eNOS but not CXCR4 in tumors subjected to combined treatment (AMD3100+L-NAME, AMD3100+1400W) were significantly greater than those in L-NAME-, 1400W- or AMD3100-treated tumors (Figs. 3-5).

Effects of treatment with agents on CXCR4, iNOS and eNOS protein expression. The expression levels of CXCR4, iNOS and eNOS in vehicle-treated tumors on western blot analysis were compared with those in tumors treated with each antagonist and inhibitor. CXCR4 expression decreased in tumors treated with 1400W, AMD3100+L-NAME, or AMD3100+1400W. The decrease in iNOS expression was greatest in tumors treated with $1400 \mathrm{~W}$. However, the expression of eNOS did not differ significantly among the treatment groups (Fig. 6).

Increased apoptosis induction by treatment with agents. When apoptosis induction in tumor parenchyma was examined by the TUNEL method, apoptotic cancer cells identified 
A

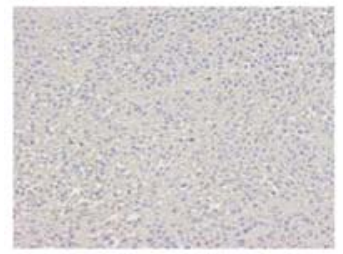

control

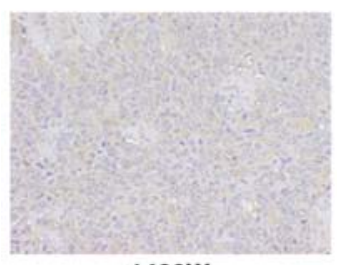

$1400 W$
CXCR4

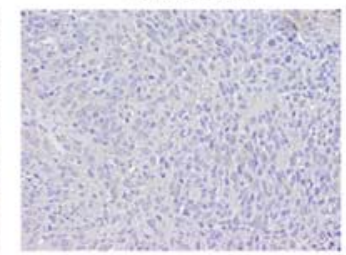

AMD3100

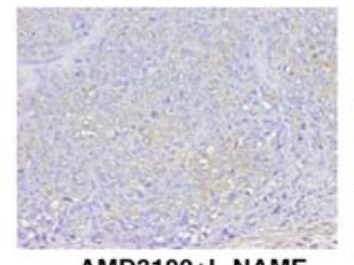

AMD3100+L-NAME

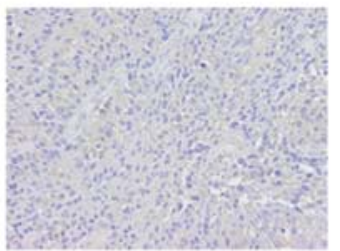

L-NAME

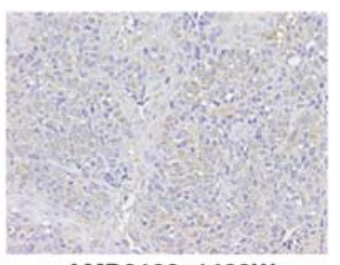

AMD3100+1400W

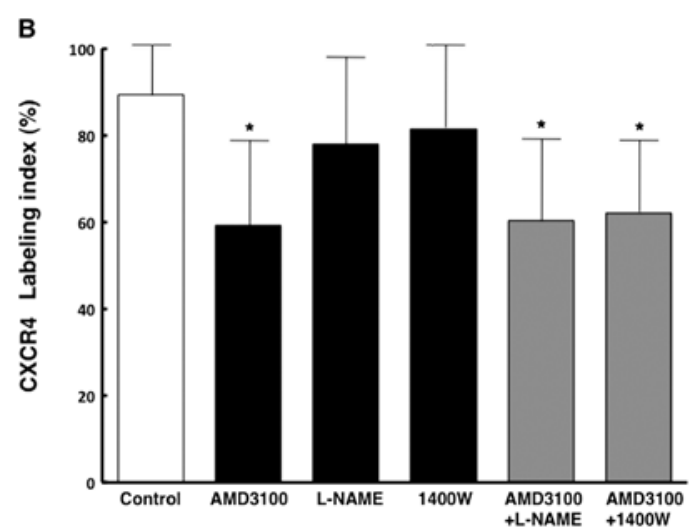

Figure 3. (A) Immunohistochemical study of CXCR4 in xenotransplanted tumors. (B) The LI of CXCR4 was obtained by calculating the ratio of positive cells to the total number of tumor cells counted in well-labeled areas, as determined by scanning twenty areas at $\mathrm{x} 200 \mathrm{magnification}$. " $\mathrm{p}<0.05$.

A

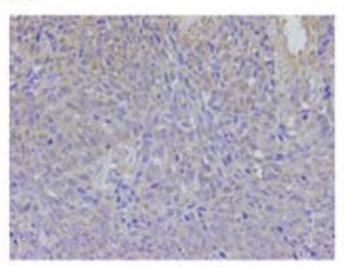

control

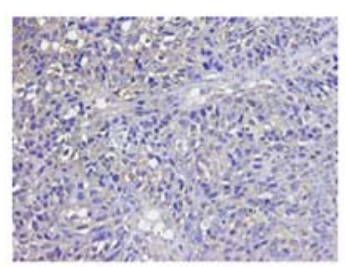

1400W
iNOS

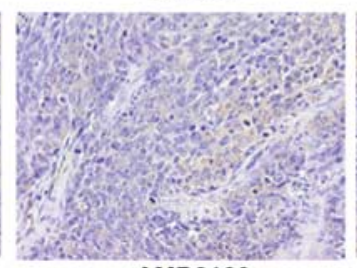

AMD3100

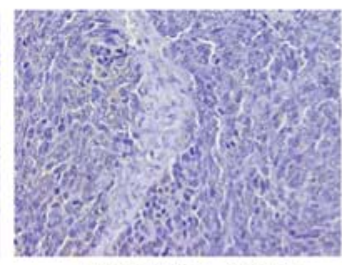

AMD3100+L-NAME

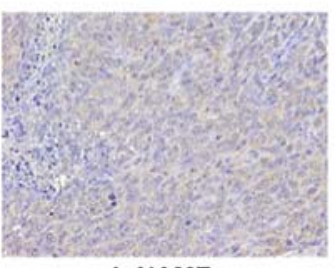

L-NAME

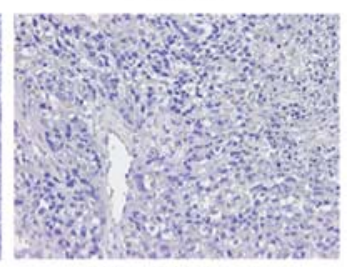

AMD $3100+1400 W$

Figure 4. (A) Immunohistochemical study of iNOS in xenotransplanted tumors. (B) The LI of iNOS in xenotransplanted tumors. ${ }^{*}$ p $<0.05,{ }^{* *}$ p $<0.005$.

A

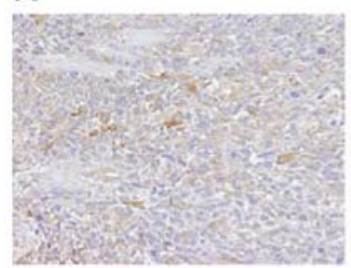

control

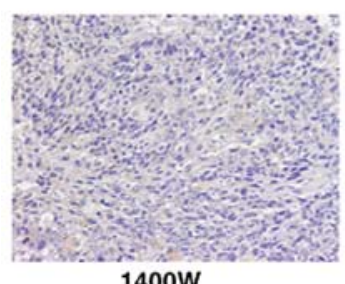

1400W

eNOS

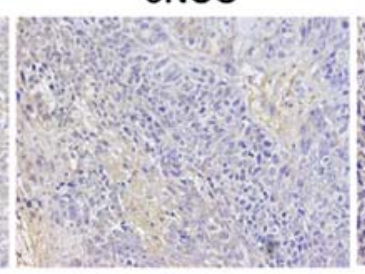

AMD3100

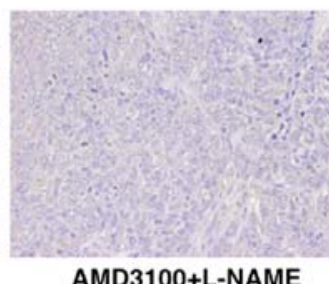

AMD3100+L-NAME

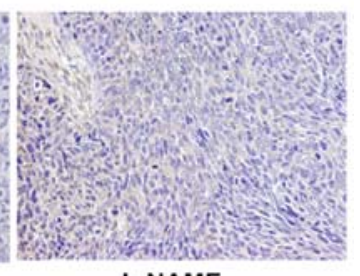

L-NAME

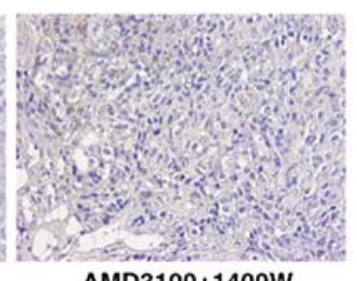

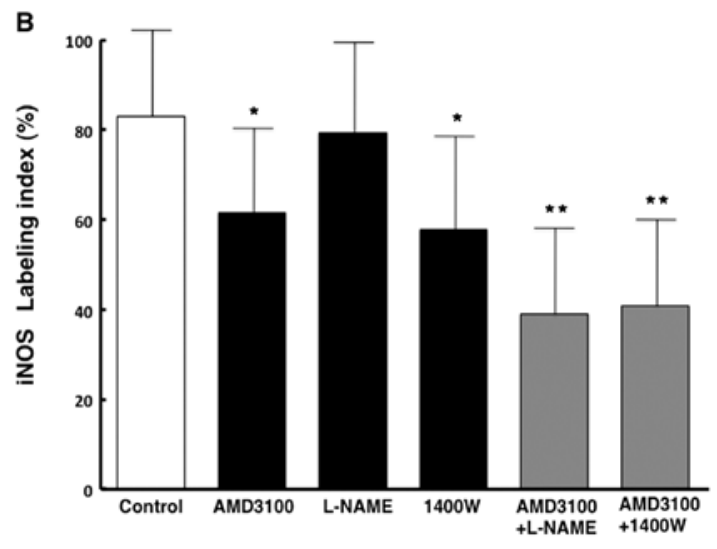

Figure 5. (A) Immunohistochemical study of eNOS in xenotransplanted tumors. (B) The LI of eNOS in xenotransplanted tumors. ${ }^{*} \mathrm{p}<0.05,{ }^{* *} \mathrm{p}<0.005,{ }^{* * *} \mathrm{p}<0.001$.

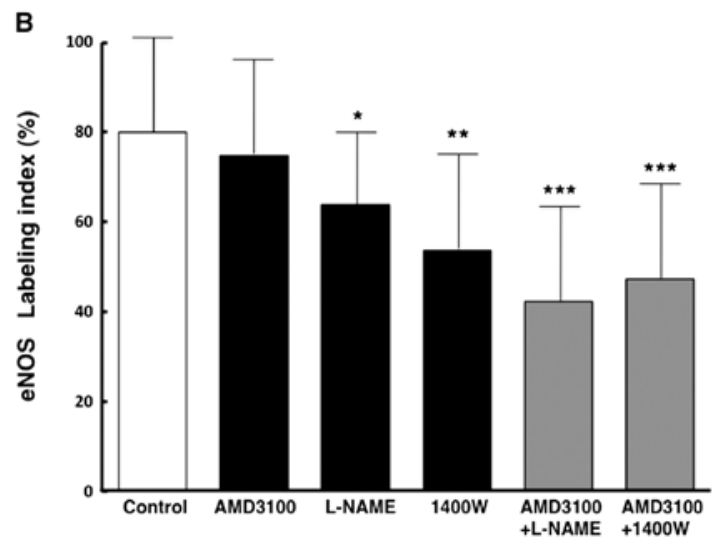




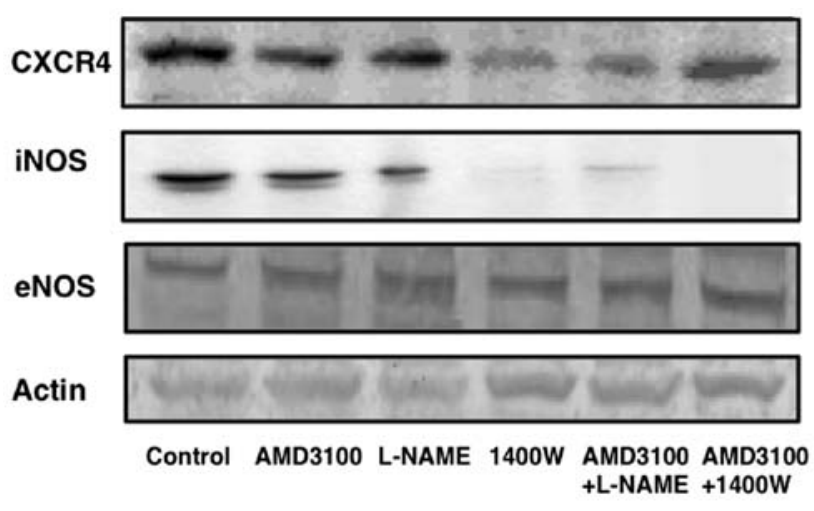

Figure 6. Expression levels of CXCR4, iNOS and eNOS in western blot analysis. by brown nuclear TUNEL signals were clearly observed in tumors treated with $1400 \mathrm{~W}$, AMD3100+L-NAME, or AMD3100+1400W. The mean apoptosis index was $0.9 \%$ in tumors treated with vehicle, but significantly increased in tumors treated with $1400 \mathrm{~W}, \mathrm{AMD} 3100+\mathrm{L}-\mathrm{NAME}$, or AMD3100+1400W (Fig. 7).

Inhibition of tumor angiogenesis by treatment with agents. When the effects of treatment with agents on tumor-induced angiogenesis in tumor stroma were examined histologically, the MVD was significantly lower in tumors treated with 1400W, AMD3100+L-NAME, or AMD3100+1400W than in vehicle-treated tumors. There was no significant difference in the MVD between $1400 \mathrm{~W}$-treated tumors and tumors

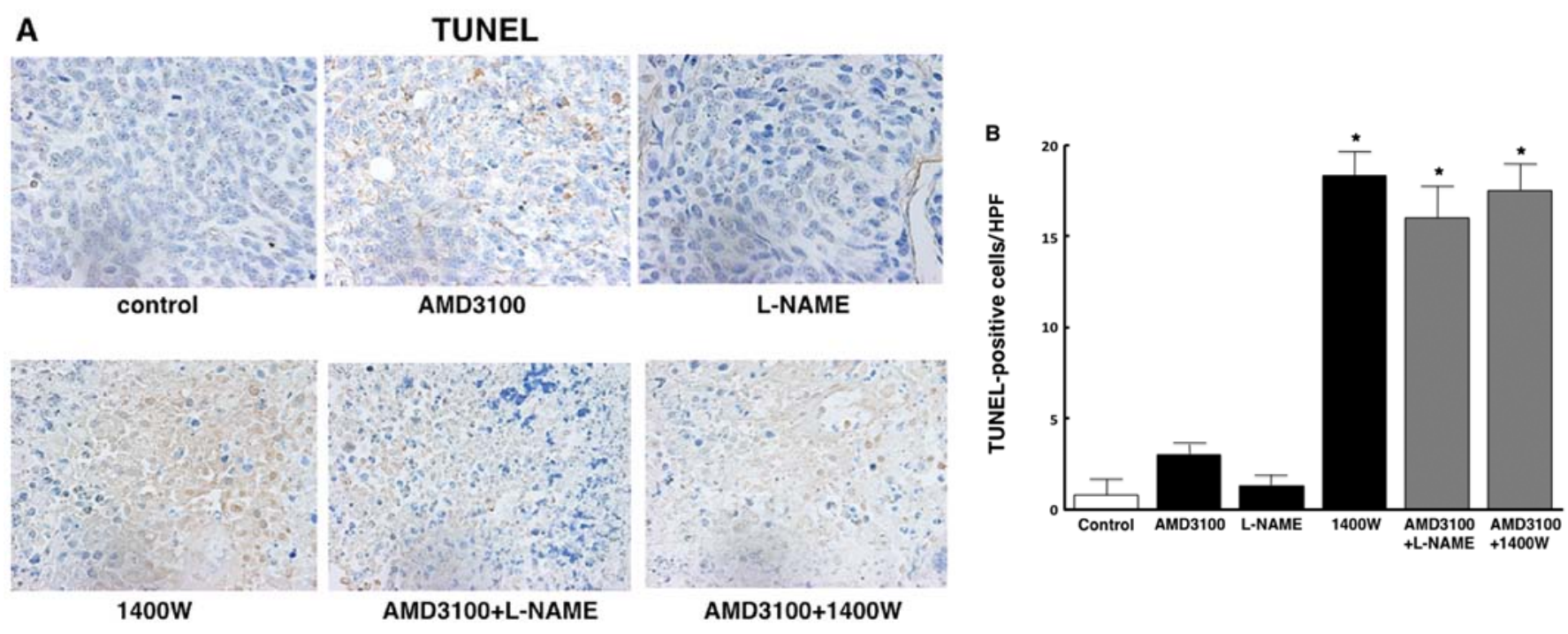

Figure 7. (A) TUNEL stain in xenotransplanted tumors. (B) TUNEL-positive cancer cells were counted in twenty areas at high magnification (x400) that shows well-labeled areas. " $\mathrm{p}<0.05$.

A

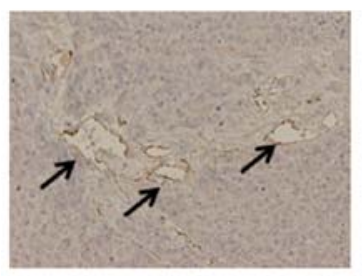

control

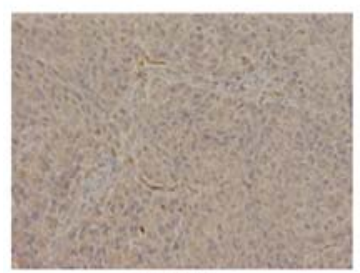

1400W

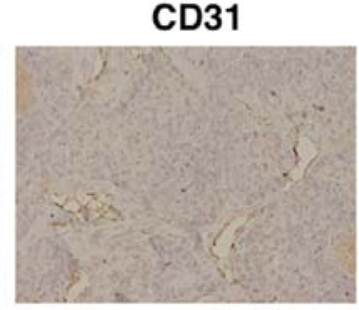

AMD3100

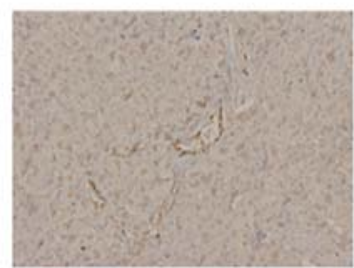

AMD3100+L-NAME

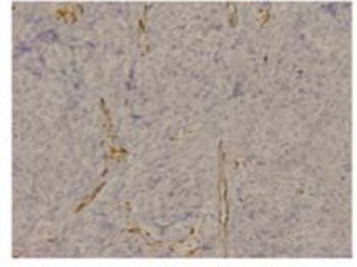

L-NAME

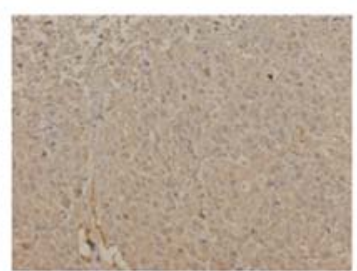

AMD3100+1400W

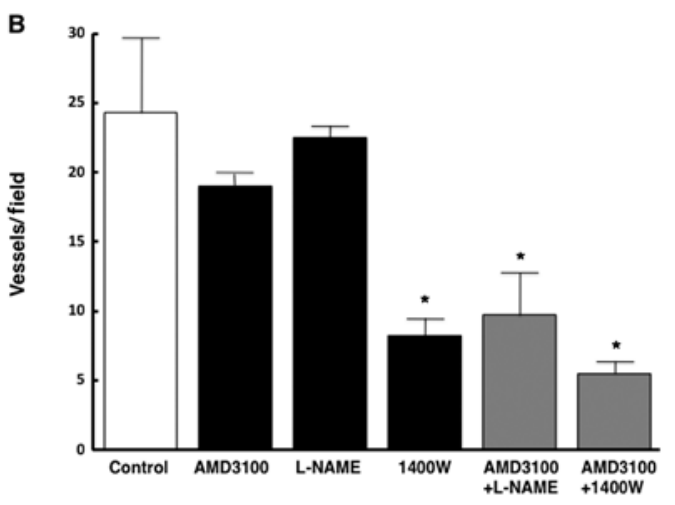

Figure 8. (A) Microvessel formation in the stroma of xenotransplanted tumors. Immunohistochemical staining for CD31 was performed to detect microvessels (arrows) in the tumor stroma (original magnification, x200). (B) Microvessel density (MVD) of each group (n=20). MVD was determined by counting the number of vessels in high-magnification fields (x200) of the tumor stroma that contained the highest number of capillaries. * $\mathrm{p}<0.05$. 
A

Agents No. of nude mice with macroscopic lung metastasis

$\begin{array}{ll}\text { Control } & 4 / 4(100 \%), \text { multiple by large nodules } \\ \text { AMD3100 } & 0 / 4(0 \%)\end{array}$

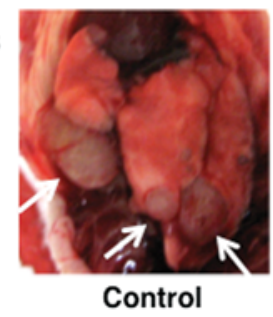

L-NAME

$4 / 4(100 \%)$, single or multiple by small nodules

\begin{tabular}{ll} 
1400W & $0 / 4(0 \%)$ \\
$\begin{array}{l}\text { AMD3100 } \\
+ \text { L-NAME }\end{array}$ & $0 / 4(0 \%)$ \\
AMD3100 & $0 / 4(0 \%)$ \\
$+1400 W$ & \\
\hline
\end{tabular}

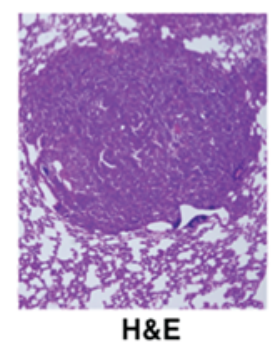

B
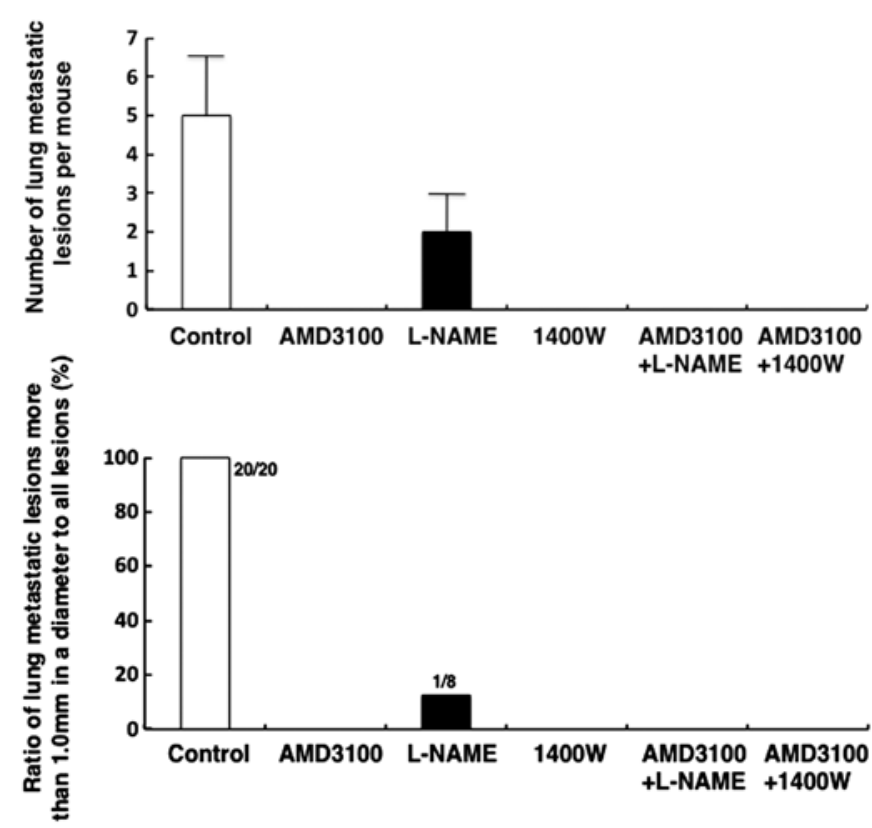

Figure 9. (A) Frequencies of macro-metastases to the lung (arrows) identified by necropsies 35 weeks after the start of treatment and a photomicrograph of metastatic lesion (original magnification, $\mathrm{x} 40$ ). (B) The number of lung metastatic lesions per mouse and the ratio of lung metastatic lesions $>1.0 \mathrm{~mm}$ in diameter to all lesions, determined by measuring 20 metastatic lesions.

treated with AMD3100+L-NAME or AMD3100+1400W (Fig. 8).

Inhibition of lung metastasis by treatment with the agents. In vehicle- and L-NAME-treated mice, metastasis to the lung occurred in all 4 mice (100\%). After treatment with AMD3100, 1400W, AMD3100+L-NAME, or AMD3100+1400W, no lung metastasis was found (Fig. 9A). The mean number of lung metastatic lesions per mouse was 5 in vehicle-treated mice and two in L-NAME-treated mice. The diameters of all lung metastatic lesions (20 lesions) in vehicle-treated mice was $\geq 1.0 \mathrm{~mm}$. Only one in eight metastatic lesions was $>1.0 \mathrm{~mm}$ in diameter in L-NAME-treated mice (Fig. 9B).

\section{Discussion}

A link between iNOS and cancer development and progression has been proposed, based on both clinical and experimental evidence. However, results have varied considerably among studies, depending on the experimental model used $(4,28,29)$. Continuous inhibition of iNOS by the selective inhibitor $1400 \mathrm{~W}$ has been shown to suppress the growth of human colon cancers as well as murine breast cancers that endogenously express iNOS (30). However, 1400W failed to inhibit murine colon cancers that did not express iNOS at appreciable levels (30). Thus, clinical as well as in vitro and in vivo evidence supports the hypothesis that targeted inhibition of iNOS and iNOS-derived NO may be an effective therapeutic approach for tumors that express iNOS. In the present study, treatment with $1400 \mathrm{~W}$ much more effectively inhibited the growth of subcutaneously xenotransplanted tumors than did treatment with L-NAME, most likely because iNOS protein expression reached appreciable levels and iNOS expression was suppressed by treatment with $1400 \mathrm{~W}$.

There are several potential mechanisms by which iNOS inhibitors can inhibit tumor growth, either directly or by sensitizing cells to other forms of stress, such as hypoxia or ROS-mediated stress. Interference with PI3K/AKT-mediated overexpression of survivin is one mechanism by which iNOS inhibition may affect the resistance of cancer cells to apoptosis $(31,32)$. Indeed, we observed that TUNEL-positive cells increased in $1400 \mathrm{~W}$-treated tumors, suggesting that iNOS inhibition might enhance the susceptibility of cancer cells to apoptosis. NO has well-established pro-angiogenic properties and stimulation of angiogenesis has been proposed as one mechanism by which iNOS expression may support tumor growth $(33,34)$. We observed a nearly 3 -fold decrease in tumor microvessel density after treatment with $1400 \mathrm{~W}$. Since both vascularization suppression and increased apoptosis can lead 
to a diminished overall rate of tumor growth, these factors may have acted additively or synergistically to cause the observed significant decrease in tumor growth in vivo. Although treatment with L-NAME or AMD3100 alone did not suppress tumor growth, combination treatment with AMD3100+L-NAME or AMD3100+1400W produced significant inhibition, comparable to that obtained by treatment with $1400 \mathrm{~W}$ alone. These results suggested that targeted inhibition of iNOS and iNOSderived NO by treatment with $1400 \mathrm{~W}$ alone and combination treatment with AMD3100+L-NAME or AMD3100+1400W induce apoptosis and significantly inhibit tumor-induced angiogenesis and proliferation of ACCIM in vivo. L-NAME is a non-selective NOS inhibitor that inhibits not only iNOS, but also eNOS. Ying and Hofseth (8) reported that although iNOS remains a viable candidate for cancer prevention and treatment, targeting eNOS might also be a viable strategy or at least deserves attention. They also reported that eNOS inhibits apoptosis and promotes angiogenesis, tumor cell proliferation, mobility and invasiveness (8).

Metastasis occurs in an organ-specific and highly organized manner. Tumors metastasize to preferred sites by diverse determinants (35) and increasing evidence has shown that the microenvironment can modulate metastatic potential (36). In our previous study, we immunohistochemically examined expression of CXCR4 in surgical specimens of ACC and our results suggested that $\mathrm{CXCR} 4$ and metastatic potential are closely related in ACC (23). Yasuoka et al $(37,38)$ reported that CXCR4 expression may be regulated by NO in breast cancer and papillary thyroid carcinoma cell lines. Our present study showed that CXCR4 expression decreased in tumors treated with $1400 \mathrm{~W}$ on western blot analysis and that treatment with AMD3100 or 1400W significantly inhibited lung metastasis. Treatment with L-NAME reduced the size and number of lung metastases as compared with treatment with vehicle. Our results suggest that CXCR4 and iNOS blockade inhibits lung metastases from ACCIM. CXCR4 and iNOS may thus be important prognostic factors for long-term survival in ACC.

\section{Acknowledgements}

This study was supported by JSPS KAKENHI grant no. 21592549 (to K.T.) and Grant-in-Aid for Young Scientists (B) 23792399 (to E.S.).

\section{References}

1. de Kerviler E, Bely N, Laccourreye O, Clement O, Halimi P and Frija G: The aryepiglottic fold as a rare location of adenoid cystic carcinoma. Am J Neuroradiol 16: 1375-1377, 1995.

2. Fordice J, Kershaw C, El-Naggar A and Goepfert H: Adenoid cystic carcinoma of the head and neck. Predictors of morbidity and mortality. Arch Otolaryngol Head Neck Surg 125: 149-152, 1999.

3. Nathan $\mathrm{C}$ and $\mathrm{Xie} \mathrm{QW}$ : Nitric oxide synthases: roles, tolls and controls. Cell 78: 915-918, 1994.

4. Fukumura D, Kashiwagi S and Jain RK: The role of nitric oxide in tumor progression. Nat Rev Cancer 6: 521-534, 2006.

5. Beckman JS, Beckman YW, Chen J, Marshall PA and Freeman BA: Apparent hydroxyl radical production by peroxynitrite: implications for endothelial injury from nitric and superoxide. Proc Natl Acad Sci USA 87: 1620-1624, 1990.

6. Prueitt RL, Boersma BJ, Howe TM, et al: Inflammation and IGF-1 activate the Akt pathway in breast cancer. Int $\mathrm{J}$ Cancer 120: 796-805, 2007.
7. Ekmekcioglu S, Ellerhorst J, Prieto VG, et al: Tumor iNOS predicts poor survival for stage III melanoma patients. Int $\mathbf{J}$ Cancer 119: 861-866, 2006.

8. Ying L and Hofseth LJ: An emerging role for endothelial nitric oxide synthase in chronic inflammation and cancer. Cancer Res 67: 1407-1410, 2007

9. Ohtsu N, Takaoka K, Segawa E, et al: Antitumor effect of inhibitors of nitric oxide synthase or cyclooxygenase-2 on human KB carcinoma cells overexpressing COX-2. Oncol Rep 24: 31-36, 2010.

10. Muller A, Homey B, Soto H, et al: Involvement of chemokine receptors in breast cancer metastasis. Nature 410: 50-56, 2001.

11. Pablos JL, Amara A, Bouloc A, et al: Stromal-cell derived factor is expressed by dendritic cells and endothelium in human skin. Am J Pathol 155: 1577-1586, 1999.

12. Aiuti A, Webb IJ, Bleul C, Springer T and Gutierrez-Ramos JC: The chemokine SDF-1 is a chemoattractant for human CD34 ${ }^{+}$ hematopoietic progenitor cells and provides a new mechanism to explain the mobilization of $\mathrm{CD} 34^{+}$progenitors to peripheral blood. J Exp Med 185: 111-120, 1997.

13. Tashiro K, Tada H, Heilker R, Shirozu M, Nakano T and Honjo T: Signal sequence trap: a cloning strategy for secreted proteins and type I membrane proteins. Science 261: 600-603, 1999.

14. Bleul CC, Fuhlbrigge RC, Cassasnovas JM, Aiuti A and Springer TA: A high efficacious lymphocyte chemoattractant, stromal cell-derived factor-1 $\alpha$ (SDF-1 $\alpha)$. J Exp Med 184: 1101-1109, 1996.

15. Campbell JJ, Hedrick J, Zlotnik A, Siani MA, Thompson DA and Butcher EC: Chemokines and the arrest of lymphocytes rolling under flow conditions. Science 279: 381-384, 1998.

16. Grabovsky V, Feigelson S, Chen C, et al: Subsecond induction of $\alpha 4$ integrin clustering by immobilized chemokines stimulates leukocyte tethering and rolling on endothelial vascular cell adhesion molecule 1 under flow conditions. J Exp Med 192: 495-506, 2000.

17. Wright N, Hidalgo A, Rodriguez-Frade JM, et al: The chemokine stromal cell-derived factor-1 $\alpha$ modulates $\alpha 4 \beta 7$ integrin-mediated lymphocytes adhesion to mucosal addressin cell adhesion molecule-1 and fibronectin. J Immunol 168: 5268-5277, 2002.

18. Ganju RK, Brubaker SA, Meyer J, et al: The $\alpha$-chemokine, stromal cell-derived factor- $1 \alpha$, binds to the transmembrane G-protein-coupled CXCR-4 receptor and activates multiple signal transduction pathways. J Biol Chem 273: 23169-23175, 1998.

19. Homey B, Muller A and Zlotnik A: Chemokines: agents for the immunotherapy of cancer? Nat Rev Immunol 2: 175-184, 2002.

20. Cardones AR, Murakami T and Hwang ST: CXCR4 enhances adhesion of B16 tumor cells to endothelial cells in vitro and in vivo via $\beta(1)$ integrin. Cancer Res 63: 6751-6757, 2003.

21. Murakami T, Maki W, Cardones AR, et al: Expression of CXC chemokine receptor- 4 enhances the pulmonary metastatic potential of murine B16 melanoma cells. Cancer Res 62: 7328-7234, 2002.

22. Robledo MM, Bartolome RA, Longo N, et al: Expression of functional chemokine receptor CXCR3 and CXCR4 on human melanona cells. J Biol Chem 276: 45098-45105, 2001.

23. Zushi Y, Noguchi K, Hashitani S, et al: Relations among expression of CXCR4, histological patterns and metastatic potential in adenoid cystic carcinoma of head and neck. Int $\mathrm{J}$ Oncol 33: 1133-1139, 2008.

24. Hashitani S, Urade M, Zushi Y, Segawa E, Okui S and Sakurai K: Establishment of nude mouse transplantable model of a human adenoid cystic carcinoma of the oral floor showing metastasis to the lymph node and lung. Oncol Rep 17: 67-72, 2007.

25. Ovejera AA, Houchens DP and Baker AD: Chemotherapy of human tumor xenografts in genetically athymic mice. Ann Clin Lab Sci 8: 50-56, 1978.

26. Hsu SM, Raine L and Franger H: Use of avidin-biotin-peroxidase complex $(\mathrm{ABC})$ in immunoperoxidase techniques: a comparison between ABC and unlabeled antibody (PAP) procedures. J Histochem Cytochem 29: 577-580, 1981.

27. Gavrieli Y, Sherman Y and Ben-Sasson SA: Identification of programmed cell death in situ via specific labeling of nuclear DNA fragmentation. J Cell Biol 119: 493-501, 1992.

28. Ekmekcioglu S, Tang C and Grimm EA: NO news is not necessarily good news in cancer. Curr Cancer Drug Targets 5: 103-115, 2005.

29. Wink DA, Ridnour LA, Hussain SP and Harris CC: The reemergence of nitric oxide and cancer. Nitric Oxide 19: 65-67, 2008. 
30. Li G, Yang T and Yan J: Cyclooxygenase-2 increased the angiogenic and metastatic potential of tumor cells. Biochem Biophys Res Commun 299: 886-890, 2002.

31. Engels K, Knauer SK, Loibl S, et al: NO signaling confers cytoprotectivity through the survivin network in ovarian carcinomas. Cancer Res 68: 5159-5166, 2008.

32. Fetz V, Bier C, Habtemichael N, et al: Inducible NO synthase confers chemoresistance in head and neck cancer by modulating survivin. Int J Cancer 124: 2033-2041, 2009.

33. Roy HK, Wali RK, Kim Y, et al: Inducible nitric oxide synthase (iNOS) mediates the early increase of blood supply (EIBS) in colon carcinogenesis. FEBS Lett 581: 3857-3862, 2007.

34. Singh RP and Agarwal R: Inducible nitric oxide synthasevascular endothelial growth factor axis: a potential target to inhibit tumor angiogenesis by dietary agents. Curr Cancer Drug Targets 7: 475-483, 2007.
35. Nguyen DX, Bos PD and Massague J: Metastasis: from dissemination to organ-specific colonization. Nat Rev Cancer 9: 274-284, 2009.

36. Joyce JA and Pollard JW: Microenvironmental regulation of metastasis. Nat Rev Cancer 9: 239-252, 2009.

37. Yasuoka H, Kodama R, Hirokawa M, et al: CXCR4 expression in papillary thyroid carcinoma: induction by nitric oxide and correlation with lymph node metastasis. BMC Cancer 8: 274 , 2008.

38. Yasuoka H, Tsujimoto M, Yoshidome K, et al: Cytoplasmic CXCR4 expression in breast cancer: induction by nitric oxide and correlation with lymph node metastasis and poor prognosis. BMC Cancer 8: 340, 2008. 